\section{Vertèbres sandwichs}

\section{Sandwich Vertebra}

\section{A.-S. Peru · M. Jourdain}

Reçu le 29 mars 2019; accepté le 10 mai 2019

(C) SFMU et Lavoisier SAS 2019

Un homme de 44 ans est amené aux urgences suite à une chute de moto, à faible cinétique, en glissant et avec un équipement de protection complet qui ne semble pas abîmé. Il s'est relevé seul et a marché pour relever sa moto avant l'arrivée des secours. Cliniquement, le patient est peu algique et se plaint surtout d'une douleur du genou qui n'est pas déformé et mobile. On retrouve une douleur diffuse lombaire sans point exquis à la pression des reliefs osseux. Il affirme être atteint d'une pathologie des os « comme la maladie des os de verre » diagnostiquée à l'âge de 20 ans suite à un accident de la route. Des radiographies des zones douloureuses nous permettent d'identifier sur les clichés des vertèbres lombaires la pathologie osseuse du patient (Fig. 1). En effet, elle met en évidence des vertèbres sandwichs caractéristiques d'une ostéopétrose bénigne ou " maladie des os de marbre », la maladie d'Albers-Schönberg [1,2]. Il s'agit d'un des neuf types d'ostéopétrose humaine. De transmission autosomique dominante, mais de pénétrance variable, elle est due à des mutations hétérozygotes sur le gène voltage-dépendant 7 (CLCN7) (13p13) codant pour un canal chlore. La maladie d'AlbersSchönberg est souvent asymptomatique ( $45 \%$ des patients) et de découverte fortuite à l'occasion d'une fracture (os paradoxalement fragile du fait de sa rigidité), d'atteintes des nerfs crâniens par compression ou d'une ostéomyélite favorisée par la maladie. La prévalence estimée en Europe est de 1/20 000. Un diagnostic génétique est possible, mais le plus souvent l'examen clinique et un bilan radiologique permettent d'affirmer le diagnostic. Cette anomalie génétique diminue l'activité ostéoclastique, créant ainsi une ostéosclérose et une condensation de l'os spongieux. On retrouve ainsi un aspect caractéristique des vertèbres avec un aspect « d'os dans l'os » des corps vertébraux, comme dans notre cas. L'ostéosclérose peut concerner aussi la base du crâne et le bassin. L'espérance de vie est normale.

\footnotetext{
A.-S. Peru $\cdot$ M. Jourdain $(\square)$

Service des urgences et SMUR, hôpital Jean-Bernard, avenue Désandrouin, BP 479, F-59322 Valenciennes cedex, France

e-mail : mat_jourdain@hotmail.com
}

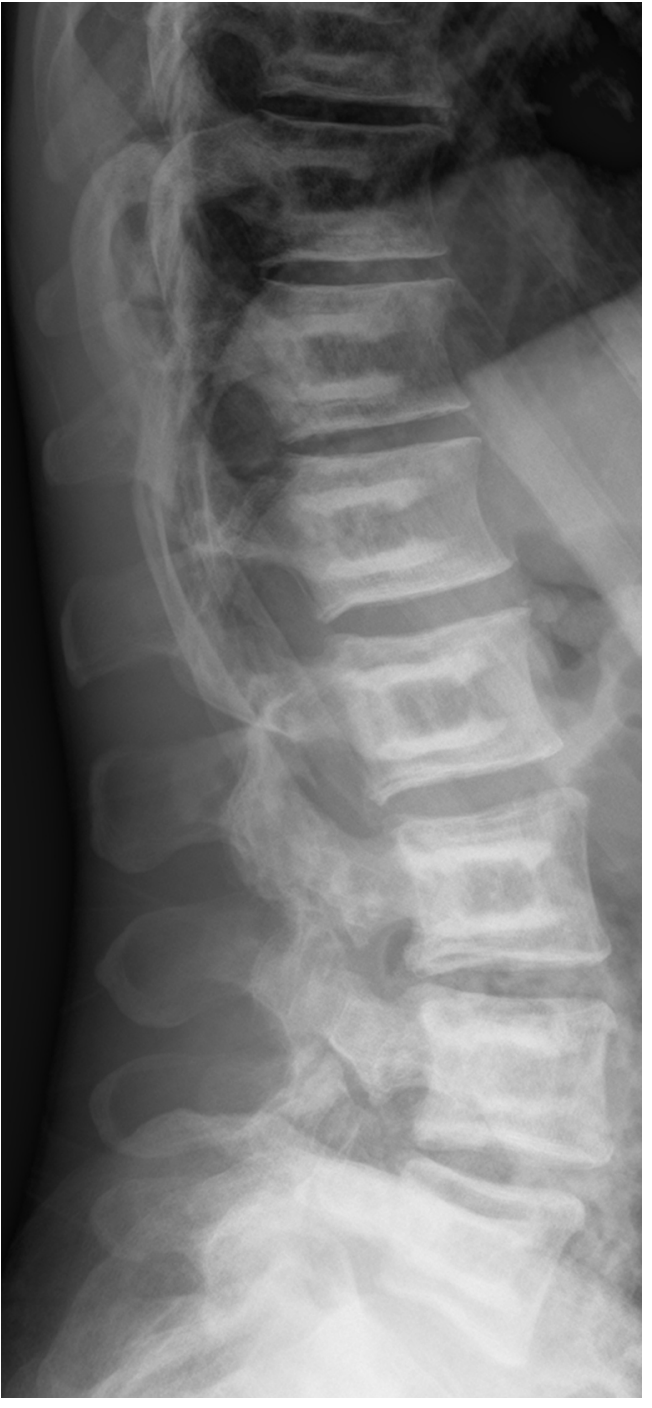

Fig. 1 Radiographie du rachis lombaire en vue de profil : image d'endostose des corps vertébraux

\section{Références}

1. De Vernejoul MC (2008) Sclerosing bone disorders. Best Pract Res Clin Rheumatol 22:71-83

2. Savarirayan R (2012) Ostéopétrose d'Albers-Schönberg. ORPHANET www.orpha.net 